\title{
App EnEM: Protótipo de Aplicativo Preparatório para o ENEM com base nos Estilos de Aprendizagem do modelo VARK
}

\section{APP ENEM: ENEM PREPARATORY APPLICATION PROTOTYPE BASED ON VARK MODEL LEARNING STYLES}

\author{
Raiza Portilho Nunes - Universidade Federal do Pará (UFPA) - \\ raisamendes20@gmail.com \\ Isadora Mendes dos Santos - Universidade Federal Rural da Amazônia (UFRA) - \\ isadoramsantos@gmail.com \\ Carlos dos Santos Portela - Universidade Federal do Pará (UFPA) - \\ carlos3mf@gmail.com
}

Resumo. O M-Learning é um paradigma educacional que permite um ambiente natural de estudos, fazendo o aluno relembrar elou memorizar conhecimentos adquiridos na escola, em qualquer lugar. No entanto, alguns desenvolvedores apenas replicam a metodologia tradicional de ensino em seus aplicativos. $O$ desenvolvimento de ferramentas que privilegiem os diversos estilos de aprendizagem tende a favorecer a eficiência do aprendizado. Nesse contexto, este trabalho objetiva apresentar um protótipo de aplicativo educativo para complementar os estudos para o Exame Nacional do Ensino Médio (ENEM), de modo que suas funcionalidades atendam a todos os estilos de aprendizagem baseado no modelo Visual, Auditivo, Leitura/Escrita e Cinestésico (VARK).

Palavras-Chave: M-learning, Aplicativo, ENEM, Vark.

Abstract. M-Learning is an educational paradigm that enables a natural study environment, allowing the student to remember and/or memorize school knowledge anywhere. However, some developers only replicate the traditional teaching methodology in their applications. The development of tools that privilege the different learning styles tend to favour the learning efficiency. In this context, this work aims to present an educational software prototype for complementary study to the Brazilian High School National Exam (ENEM), in order to its functionalities meet all learning styles based on the Visual, Auditive, Reading/Writing and Kinesthetic (VARK) model.

Keywords: m-learning, Application, ENEM, Vark.

\section{Introdução}

A evolução tecnológica impulsionou um novo paradigma educacional chamado Mobile Learning ou m-learning. Esse paradigma, que envolve o uso de dispositivo móvel, sozinho ou em combinação com outras Tecnologias Digitais de Informação e Comunicação (TDICs), pode aumentar a motivação do estudante (Franciscato e Medina, 2008). Um dos motivos que torna essa tecnologia presente no cotidiano das pessoas é a necessidade de realizar atividades com praticidade e mobilidade. 
Por intermédio do m-learning, é possível viabilizar um ambiente mais natural de estudos, sem abstrações, possibilitando ao aluno relembrar e/ou fixar conhecimentos adquiridos na escola. Em conformidade com Junior (2009), a constante necessidade de mobilização, de acesso e troca de informações em qualquer tempo e espaço, de compartilhamento de ideias, experiências e conhecimento, e a necessidade de ampliar os espaços formais de educação, favorecem o emprego da m-learning. Para Arantes e Seabra (2016), o poder ubíquo dos smartphones que possibilita ao aluno aprender em qualquer momento e lugar, tem conquistado cada vez mais adeptos, pois fornece ao usuário a informação mais atualizada possível, bem como o suporte para a aprendizagem formal e informal. Ou seja, o m-learning confere oportunidades para seus adeptos escolherem o local e forma de estudar, oportunizando os pontos fortes de seus estilos de aprendizagem.

De acordo com Mühlbeier et al. (2011), estilos de aprendizagem são denominados como a maneira que o indivíduo se concentra, processa e retém informação, ou seja, suas características pessoais e preferências são peculiaridades que intervém diretamente na maneira como irá construir novos saberes. Dentre as abordagens relacionadas aos estilos de aprendizagem existe o modelo VARK proposto por Fleming e Mills (1992). O nome VARK deriva do acrônimo em inglês de Visual, Aural, Read/Write e Kinesthetic (Visual, Auditivo, Leitura/Escrita e Cinestésico, em tradução livre para o português). Esse modelo pressupõe que a aprendizagem ocorre por meio dos sentidos visual, auditivo e cinestésico e que cada aluno possui um estilo de aprendizagem predominante.

Mühlbeier et al. (2011), informam que uma combinação inadequada entre os estilos de aprendizagem dos alunos e as metodologias pedagógicas aplicadas na escola pode ocasionar tédio e distração nos discentes, implicando em resultados deficientes desinteresse no curso. Gallert (2005), enfatiza que o desenvolvimento de ferramentas que privilegiem os diversos estilos de aprendizagem, tendem a favorecer a eficiência do aprendizado. Para a autora, o desenvolvimento de artefatos tecnológicos requer atenção às questões pedagógicas e aos estilos de aprendizagem, para que o instrumento se torne atrativo ao usuário e estimule sua vontade de aprender.

Neste sentido, um desafio a ser superado é o hábito de alguns desenvolvedores em replicar a metodologia tradicional escolar para os aplicativos educativos, sem apresentar recursos que explorem as preferências e características predominantes de seus usuários. Essa crítica é trazida por Neves, Melo e Machado (2014), em que os autores sugerem a necessidade de um olhar mais crítico para os discursos que apenas visam inserir as tecnologias móveis na escola sem nenhuma discussão sobre a proposta pedagógica, os tempos e os espaços de aprendizagem, favorecendo um uso meramente passivo desses recursos sem explorar todo o potencial que os smartphones apresentam para a apropriação criativa e para o ensino e aprendizagem para além dos espaços da sala de aula. A simples transposição de conteúdos originalmente impressos em papel para uma mídia eletrônica não traz nenhuma vantagem intrínseca do ponto de vista didático-pedagógico.

Diante do exposto, esse trabalho consiste na apresentação de um protótipo de aplicativo para o ensino complementar de conteúdos abordados no Exame Nacional do Ensino Médio (ENEM), denominado Ensino baseado em Estímulos Multi-sensoriais (App EnEM), inspirado nos estilos de aprendizagem do modelo VARK. A escolha desse modelo deve-se a possibilidade de adaptação e implementação em um ambiente hipermídia dos três principais canais receptores sensoriais: visão (figuras, imagens, animações), audição (sons) e tato (toque e movimento) (Gallert e Martins, 2005). Ressalta-se também que a escolha de adotar conteúdos preparatórios para o ENEM, deve- 
se ao fato de que o exame é amplamente utilizado como instrumento de acesso pela maioria das instituições públicas do país (Arantes e Seabra, 2016).

Além desta seção, aborda-se na Seção 2 o referencial teórico explorando o conceito dos estilos de aprendizagem VARK. A Seção 3 apresenta a metodologia de pesquisa. A Seção 4 apresenta o protótipo de telas do software proposto. E por fim, na Seção 5 são apresentadas algumas considerações sobre o trabalho realizado e propostas para trabalhos futuros.

\section{Referencial Teórico}

Nas últimas décadas, diversos estudos sobre os estilos de aprendizagem foram desenvolvidos, contudo, uma minoria destes dedicou-se a questões envolvendo a tecnologia, principalmente na realidade brasileira (Roza, 2017). Segundo análise de Neves, Melo e Machado (2014), os aplicativos ditos educativos disponíveis no mercado, carecem de uma proposta pedagógica relevante, pois prevalece nestes uma intencionalidade meramente comercial. Como bem destacado por Roza (2017), somente inserir recursos tecnológicos no processo educativo mostra-se insuficiente ao evidenciar a tecnologia em si, não dando a devida atenção ao aprendiz como sujeito do processo de aprendizagem.

Nagumo (2014), informa que, em geral, os indivíduos adquirem mais conhecimento em contextos informais e que as pessoas empregam naturalmente seus smartphones nas práticas de estudo, explorando os recursos para realizar suas atividades pedagógicas. Valorizando a personalização do ensino, a tecnologia promove um aprendizado que prestigia cada perfil de estudante (Nascimento et al. 2017). Os aplicativos, quando providos de distintos recursos para apresentar o conteúdo didático, podem ser bem aceitos por todos os perfis de usuários, pois os recursos tecnológicos possibilitam a adoção de diferentes teorias para solucionar problemas e assimilar conteúdo. Essas teorias são chamadas de Estilos de Aprendizagem.

De modo geral, estilos de aprendizagem referem-se à maneira como os indivíduos interagem para observar o ambiente, captar informações, processar e compreender seu significado e então transformá-las em conhecimento (Ferreira et al. 2017). Segundo Gallert (2005), planejar softwares considerando os estilos de aprendizagem individuais, viabiliza possibilidades diferenciadas de estudo e uma maior motivação para os usuários. Roza (2017) corrobora com essa perspectiva e afirma que para que as tecnologias satisfaçam seu papel de aliadas da educação, é preciso considerar as diferenças individuais de seus usuários, ou seja, seus estilos de aprendizagem.

Com a intenção de operacionalizar a concepção sobre estilos de aprendizagem através da predileção pessoal por estímulos sensoriais, Fleming e Mills (1992), desenvolveram o modelo VARK que são preferências sensoriais dos seres humanos usadas para reter informações. Em linhas gerais, o inventário VARK pressupõe que o aprendizado ocorre por meio dos sentidos visual, auditivo e tátil e que existem indivíduos que apresentam somente um estilo sensorial, predominante para obter conhecimento e há indivíduos que apresentam uma mistura equilibrada de dois ou mais estilos.

Baseando-se nas categorias sensoriais apresentadas, o modelo VARK se dispõe a identificar o estilo predominante do indivíduo e recomendar métodos personalizados para melhorar a efetividade do aprendizado para cada um dos perfis (França et al. 2016). Silva e Silva (2006), exemplificam que, se uma pessoa aprende melhor ouvindo uma explicação, então ela possui o estilo de aprendizagem predominantemente auditivo, 
embora isso não implique ser este o seu único canal de aprendizado, pois tal pessoa pode ser parcialmente visual, visto que aprende visualizando a matéria e pode, ainda, ser parcialmente cinestésico se consegue aprender através de atividades interativas.

Entender como os estímulos se comportam em cada pessoa, pode tornar o ensino mais proveitoso e melhorar o desempenho dos estudantes (Ferreira et al. 2017). Nessa concepção, os quatro estilos de aprendizagem descritos na teoria VARK são (Fleming e Mills, 1992):

- Visual (V) - aprendem melhor visualmente e preferem as informações provindas por demonstrações visuais e descrições. Gostam de utilizar listas esquemas, figuras, gráficos e outros recursos visuais para manter o raciocínio e organizar seus pensamentos. São distraídas pelos movimentos ou ações.

- Auditivo (A) - memorizam mais as informações transmitidas por meio da fala. $\mathrm{O}$ aluno que possui estilo auditivo aprende melhor por sons, pode facilmente se distrair e desvia a atenção com outros sons que envolvem o ambiente de sala de aula.

- Leitura e Escrita (R) - preferem estudar isoladamente, presos a textos de leituras. Outra característica de alunos com este estilo é que possuem facilidades com a escrita, desenvolvem melhor trabalhos que necessitem a busca por livros, se dão bem com conceitos teóricos e preferem deixar formalizados seus conhecimentos no papel.

- Cinestésico $(\mathrm{K})$ - preferem trabalhos em grupo e possuem como principal característica a aprendizagem por meio de experiências sobre o objeto de estudo. Além disso, possuem pré-disposição pela colaboração entre os agentes envolvidos no processo de aprendizagem. Aulas práticas são preferidas por alunos com esse estilo.

Gallert e Martins (2005), consideram que a implementação de um sistema que apresente o mesmo conteúdo através de diferentes estímulos sensoriais favorece todos os tipos de aprendizes de acordo com o modelo VARK. Assim, se o usuário apresenta estilo visual e leitura/escrita, poderá assimilar o conteúdo com mais figuras, imagens e animações do que texto. O aprendiz auditivo vai preferir escutar a gravação do conteúdo. E o cinestésico vai ser beneficiado pela mobilidade que um aplicativo em um smartphone pode proporcionar, além de usufruir de imagens e animações que simulam o movimento.

Nesse sentido, pode-se compreender que quanto mais habilidades o indivíduo tiver desenvolvido, maior será a facilidade de lidar com as diferentes abordagens educativas por ele vivenciadas (Mühlbeier e Mozzaquatro, 2011).

\section{Metodologia}

A caracterização metodológica deste trabalho qualifica-se como aplicada, quanto à sua natureza, motivada pela necessidade de adquirir conhecimento para aplicação de seus resultados, considerando que para sua elaboração foi realizada uma pesquisa bibliográfica a fim de obter o embasamento necessário para o entendimento da pesquisa, fundamentando-se nas teorias existentes, sendo estas, obtidas da literatura e trabalhos semelhantes.

Em seguida, foi feito o levantamento de requisitos para o projeto, usando como base as categorias sensoriais apresentadas no modelo VARK. Por fim, a última etapa consistiu em desenvolver o protótipo de telas do aplicativo. Para a construção do 
protótipo, foi utilizado o software Justinmind Prototyper ${ }^{l}$, onde é possível criar protótipos funcionais de alto nível para dispositivos móveis. Esse software está disponível em uma versão gratuita, contendo algumas limitações, e na versão paga, que é a licença completa da aplicação. Neste trabalho, optou-se por trabalhar com a versão gratuita por restrições orçamentárias dos autores/pesquisadores.

\section{Protótipo do Aplicativo Desenvolvido}

Caracterizado por uma usabilidade simples e intuitiva, o aplicativo proposto foi inspirado nos diferentes estilos de aprendizagem já descritos nesse trabalho. Além de facilitar a aprendizagem através de uma interface agradável, os recursos para leituras, sons, imagens, gráficos e vídeos possibilitam a concepção de um ensino multissensorial e multidisciplinar, pois a ferramenta disponibiliza conteúdos didáticos de todas as áreas de conhecimento abordadas no ENEM. A Figura 1 ilustra a tela inicial do aplicativo App EnEM.

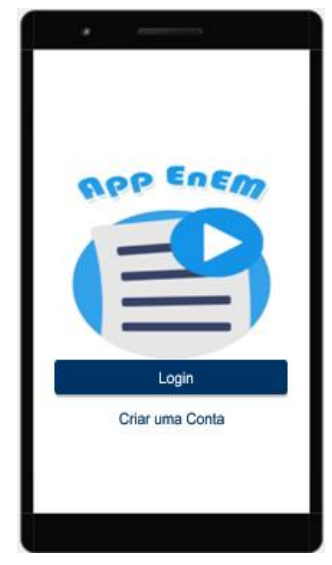

Figura 1 - Tela Inicial do aplicativo.

Após validar seu acesso na tela de Login, o usuário acessa a tela onde estarão disponíveis os conteúdos didáticos. A opção Simulados, visualizada na Figura 2, disponibiliza as provas completas do exame desde 2009. As demais opções apresentam conteúdos e exercícios específicos para cada disciplina da referida área.

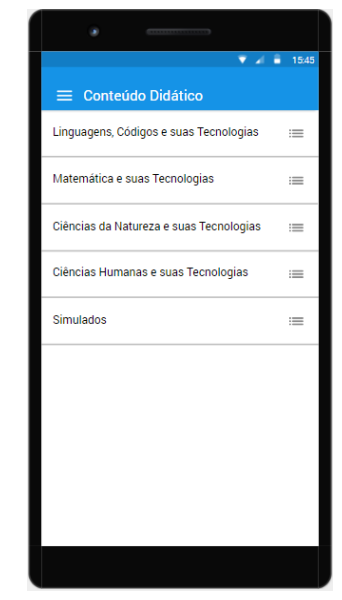

Figura 2 - Menu Inicial.

Caso selecione uma das áreas de conhecimento disponibilizadas no menu, o usuário deverá escolher a opção desejada entre as disciplinas que compõem a área

\footnotetext{
${ }^{1}$ https://www.justinmind.com
} 
escolhida. Para exemplificar, a Figura 3 - A representa as disciplinas que compõem a área de Ciências Humanas e Suas Tecnologias. Ao clicar na disciplina desejada, o aprendiz poderá estudar através das diversas funcionalidades que o aplicativo oferece, como exposto na Figura 3 - B. Ele pode escolher entre ler o conteúdo, assistir ao vídeo ou escutar o áudio dos conteúdos listados. É interessante ressaltar que Gallert (2005), expressa que o desenvolvimento de ferramentas, que valorizam os diversos estilos de aprendizagem, implica em eficiência do processo de aprendizagem, além de tornar a ferramenta mais interessante para o usuário.

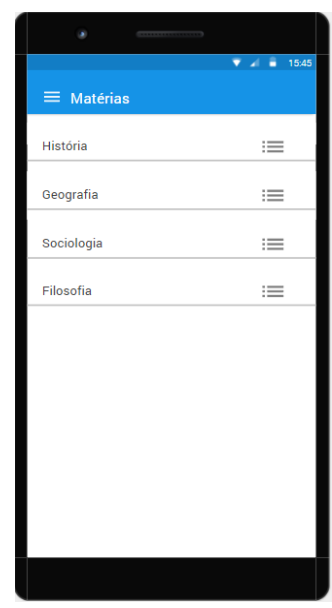

A) Disciplinas

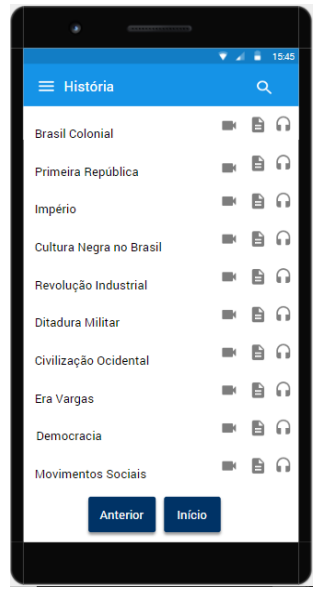

B) Conteúdo Multissensorial

Figura 3 - Telas de Conteúdo Didático.

Os recursos para leitura são propícios para indivíduos com estilo visual e leitura/escrita, sendo necessário tornar o ambiente de aprendizagem mais adequado a esses aprendizes através de textos com ilustrações e resumos para auxiliar o aprendiz a representar visualmente conceitos abstratos (Filatro, 2014). Gallert (2015), exprime que a disponibilização de vídeos é interessante para proporcionar uma melhor visibilidade do tema estudado, pois favorece a memorização e organização do conhecimento. Além disso, um vídeo apresenta aulas explicativas, agradáveis a aprendizes visuais e a possibilidade de aprendizes auditivos ouvirem uma aula (França et al., 2016).

Ao optar por ler o conteúdo desejado, o usuário terá a sua disposição textos ilustrados, como apresentado na Figura $4-$ A, com figuras, cartuns e tirinhas em quadrinhos para tornar o aprendizado mais agradável e estimulante, principalmente para aprendizes visuais. Em geral, aprendizes visuais gostam de olhar o que estão aprendendo, portanto, textos com ilustrações ajudam o indivíduo visual a entender melhor as ideias e informações apresentadas (Gallert e Martins, 2005).

O aplicativo possibilita ao usuário copiar a parte do texto que considerar interessante para inserir em um bloco de notas localizado no menu lateral. Além disso, como ilustrado na Figura 4 - B, é possível marcar ou sublinhar textos disponibilizados no aplicativo. Tais recursos, são interessantes para aprendizes com predominância do estilo de aprendizagem leitura/escrita, visual e cinestésico.

Filatro (2014), ressalta a importância de proporcionar o uso de marcadores para enfatizar pontos interessantes no texto, estimulando o aprendiz a interagir com materiais escritos. Segundo Gallert (2005), a leitura em material digital é mais lenta que em material impresso, sendo necessário o uso de marcadores de texto para prender a atenção do 
usuário. Além disso, o texto precisa ser claro e objetivo. Ao reunir esses componentes (marcadores, texto claro e objetivo) é possível ampliar a atenção do aprendiz em 124\%.

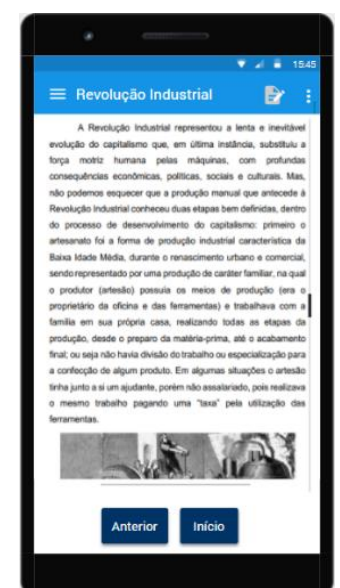

A) Texto com Ilustração.

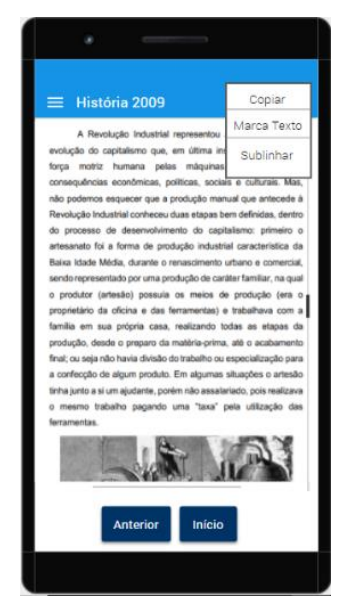

B) Destacar Texto.

Figura 4 - Textos Ilustrados.

$\mathrm{Na}$ Figura 5 - A é possível observar que o usuário poderá criar seu próprio exercício, especificando a quantidade de questões, assunto, disciplina e ano. A disposição de exercícios personalizados e simulados completos do ENEM são recursos que valorizam os estilos de aprendizagem leitura/escrita, além de envolver o aprendiz cinestésico na aprendizagem, pois, tais indivíduos possuem preferência por executar/fazer atividades propostas nos exercícios (Gallert, 2005).

As questões disponíveis no aplicativo são provenientes das provas do ENEM de anos passados, disponibilizadas no site oficial do exame ${ }^{2}$ e apresentam o mesmo enunciado e quantidade de alternativas do exame, como observado na Figura 5 - B.

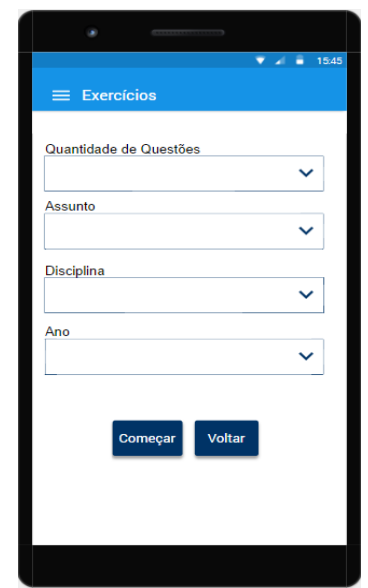

A) Exercícios Personalizados

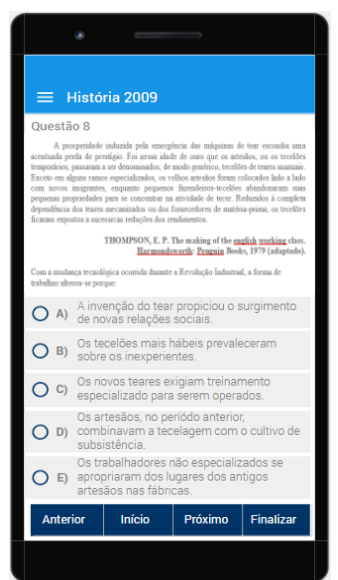

B) Questões do ENEM.

Figura 5 - Telas de Exercícios.

Ao finalizar um simulado, um feedback de desempenho é exibido na tela contendo seu número de acertos, erros e tempo total. Além disso, o aprendiz poderá visualizar seu feedback graficamente como visto na Figura 6. Gallert (2005), informa que gráficos são interessantes para aprendizes visuais, que em geral têm preferência por formas simbólicas

\footnotetext{
${ }^{2}$ http://portal.inep.gov.br/provas-e-gabaritos
} 
de representação de informação, permitindo-lhe uma melhor visibilidade do conteúdo estudado e a assimilação do conhecimento pode ser favorecida por estes recursos.

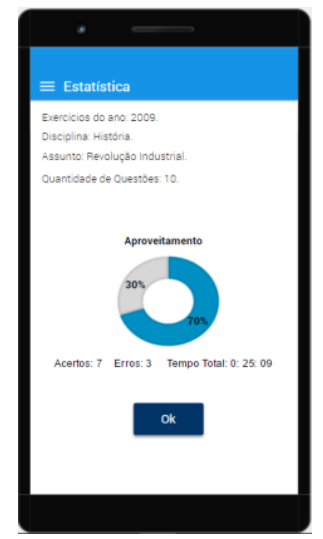

Figura 6 - Gráfico de Desempenho

O botão de navegação Menu Lateral, localizado no canto superior esquerdo, levará o usuário a outras funcionalidades do aplicativo, como o Bloco de Notas, onde o usuário poderá criar qualquer anotação que julgar interessante para seu aprendizado. Esse recurso favorece aprendizes com estilo visual, leitura/escrita e cinestésico. Segundo Schmitt e Domingues (2016), o estilo de aprendizagem leitura/escrita é característico de indivíduos tomadores de notas, que durante atividades como palestras e leitura de materiais complexos, fazem anotações, desenham planos e esquemas para lembrar os conteúdos. Além disso, aprendizes visuais e cinestésicos também utilizam esse tipo de recurso em suas atividades, sendo recomendável tornar o ambiente de aprendizagem mais oportuno às pessoas predominantemente visuais, por meio de espaços em branco para que esses aprendizes façam anotações e indivíduos cinestésicos consigam experimentar um ambiente de interação com o objeto de estudo, pois quando estão estudando, os aprendizes cinestésicos costumam ler em voz alta suas anotações (Filatro, 2014).

Além disso, o aprendiz poderá criar ou gerar automaticamente uma Agenda de Estudos. Esse recurso é interessante para indivíduos visuais, pois oferece a possibilidade de visualizar o percurso de estudo escolhido no formato de fluxograma, que é um dos recursos utilizados no aprendizado de pessoas com estilo de aprendizagem visual (Gallert, 2005). A opção Dicas ENEM, apresenta informações úteis acerca do exame, como assuntos mais frequentes, dicas para redação e como organizar e aproveitar melhor o tempo de prova. Outras opções como Configurações, Instruções de Uso e Sobre, também se encontram nesse menu apresentado na Figura 7.

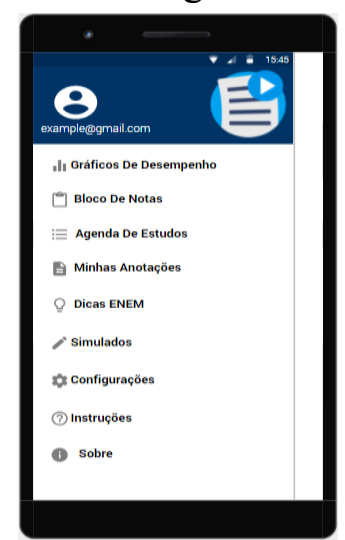

Figura 7 - Menu Lateral do aplicativo. 


\section{Considerações Finais}

Em conformidade com Mühlbeier et al. (2011), diagnosticar o estilo de aprendizagem de um indivíduo é uma tarefa complexa, que necessita de análises aprofundadas para que possa ser oferecida ao aprendiz uma experiência de aprendizagem individualizada, apresentando-lhe a informação de maneira personalizada e direcionada. A complexidade de identificar qual o estilo de aprendizagem de cada estudante, pode ser um obstáculo para a efetivação do aprendizado. Por esse motivo, um software educativo, que contemple metodologias distintas capaz de abordar todos os estilos de aprendizagem pode ser uma alternativa eficaz para potencializar o aprendizado dos estudantes. Sendo assim, este trabalho apresentou um projeto de aplicativo educativo denominado App EnEm (Ensino baseado em Estímulos Multi-sensoriais) inspirado nos diferentes estilos de aprendizagem do modelo VARK de Fleming e Mills (1992).

Como apresentado neste trabalho, é necessário conhecer o comportamento das pessoas, suas preferências, hábitos e estilo de aprendizagem predominante, para então desenvolver e propagar ferramentas digitais que promovam um aprendizado eficaz em seus usuários, acolhendo e incorporando estratégias que abarquem os diferentes estilos de aprendizagem, pois compreende-se que educação de qualidade é aquela que atende às necessidades de cada pessoa, valorizando os distintos estilos de aprendizagem e propicia condições para o aprendiz atingir seus objetivos pessoais.

Através de uma interface agradável e de fácil manipulação, os recursos de som, imagem, leitura e vídeo possibilitaram o planejamento de um aplicativo rico em apresentação de conteúdo didático, capaz de atender a indivíduos de todos os estilos de aprendizagem. Espera-se, com o desenvolvimento deste trabalho, colaborar para que as metodologias educativas sejam aliadas à tecnologia móvel, seguindo procedimentos didáticos eficazes para melhor satisfação dos usuários.

Como parte complementar a este projeto, está em andamento a validação e feedback com usuários finais, através de formulário ${ }^{3}$ elaborado pelos autores, para que se inicie a implementação. Após o desenvolvimento do aplicativo, devem ser realizados outros testes de usabilidade e experiência de uso com os usuários de diferentes perfis de aprendizagem, a fim de validar e comparar a efetividade do mesmo e aplicação em contexto educacional.

\section{Referências}

ARANTES, H. F. e SEABRA, R. D. TME: Aplicativo M-Learning para o Estudo de Conceitos Matemáticos com Ênfase no ENEM. In: Anais do XXVII Simpósio Brasileiro de Informática na Educação (SBIE 2016). DOI: 10.5753/cbie.sbie.2016.1.

FERREIRA, L.; FERRARI, M. G.; PAIVA, M. Z. P. e RODRIGUES, J. JR. Métodos de classificação aplicados à detecção automática de Estilos de Aprendizagem em um ambiente real de ensino. In: Anais do XXVIII Simpósio Brasileiro de Informática na Educação (SBIE 2017). Doi: 10.5753/cbie.sbie.2017.1517.

FILATRO, A. Estilos de Aprendizagem. In: Repositório ENAP Escola Nacional de Administração Pública. 2014.

FLEMING, N. D. e MILLS, C. Not Another Inventory, Rather a Catalyst for Reflection. In: To Improve the Academy, v. 11, 1992. Cap. 1, p. 137.

\footnotetext{
${ }^{3}$ https://forms.gle/NWTysVcd77mfdqnE8
} 
FRANÇA, C.; CUNHA, A.; ADJARDE, D. e ALAN, F. Uma Investigação sobre Estilos de Aprendizagem e Hábitos de Estudo de Engenheiros de Software. In: XXX Simpósio Brasileiro de Engenharia de Software (SBES 2016).

FRANCISCATO, F. D. e MEDINA, R. D. M-Learning e Android: um novo paradigma?. In: Revista Novas Tecnologias na Educação RENOTE, V. 6 Nº 2, Dezembro, 2008.

GALLERT, C. S. Sistema hipermídia para ensino baseado nos estilos de aprendizagem. 2005. 1-19p. Dissertação (Mestrado em Ciências da Computação). Universidade Federal de Santa Catarina. Santa Catarina-SC, 2005.

JUNIOR, P. G. G. Aprendizagem Com Mobilidade (M-Learning) Nos Processos de Ensino e de Aprendizagem: Reflexões e Possibilidades. In: Revista Novas Tecnologias na Educação RENOTE, V. 7 Nº 1, Julho, 2009.

GALLERT, C. S. e MARTINS, L. H. Sistema Hipermídia Baseado no Estilo de Aprendizagem VAK. In: VII Simpósio Internacional de Informática Educativa SIIE05. Leiria, Portugal, 16-18 Novembro de 2005.

MÜHLBEIER, A. R. e MOZZAQUATRO, P. M. Estilos e estratégias de aprendizagem personalizadas a alunos das modalidades presenciais e a distância. Revista Novas Tecnologias na Educação RENOTE, v. 9, n. 1, 2011.

MÜHLBEIER, A. R.; OLIVEIRA, L. C.; MOZZAQUATRO, P. M. e MEDINA, R. D. Análise Comprobatória teórico-prática da semelhança entre dimensões para detecção de Estilos de Aprendizagem impactando na ausência de resultados significativos. In: Revista Novas Tecnologias na Educação RENOTE, V. 9 № 2, dezembro, 2011.

NAGUMO, E. O Uso do Aparelho Celular dos Estudantes na Escola. 2014. 100f. Dissertação (Mestrado em Educação (PPGE) da Faculdade de Educação (FE)) Universidade de Brasília. 2014.

NASCIMENTO, P.; BARRETO, R.; PRIMO, T.; GUSMÃO, T. e OLIVEIRA, E. T. H. Recomendação de Objetos de Aprendizagem baseada em Modelos de Estilos de Aprendizagem: Uma Revisão Sistemática da Literatura. In: Anais do XXVIII Simpósio Brasileiro de Informática na Educação (SBIE 2017), Recife, p. 213-222.

NEVES, B. G.; MELO, R. S. e MACHADO, A. F. Universo Móvel: Um Aplicativo Educacional Livre Para Dispositivos Móveis. In: Texto Livre Linguagem e Tecnologia. Ano: 2014 - Volume: 7 - Número: 1.

ROZA, R. H. Estilos de Aprendizagem e o Uso das Tecnologias da Informação e Comunicação. 2017.157p. Tese (Doutorado em Psicologia como Profissão e Ciência) Pontíficia Universidade Católica de Campinas, Centro de Ciências da Vida, Programa de PósGraduação, Campinas, 2017.

SCHIMITT, C. da S.; e DOMINGUES, M. J. C. de S. Estilos de aprendizagem: um estudo comparativo. In: Avaliação, Campinas; Sorocaba, SP, v. 21, n. 2, p. 361-385. 2016.

SILVA, W. M.; e SILVA, E. C. L. de. Investigação dos Dados sobre Estilos de Aprendizagem dos Alunos frequentadores da base de apoio ao Aprendizado Autônomo. In: Revista Científica da UFPA, 2006. 